
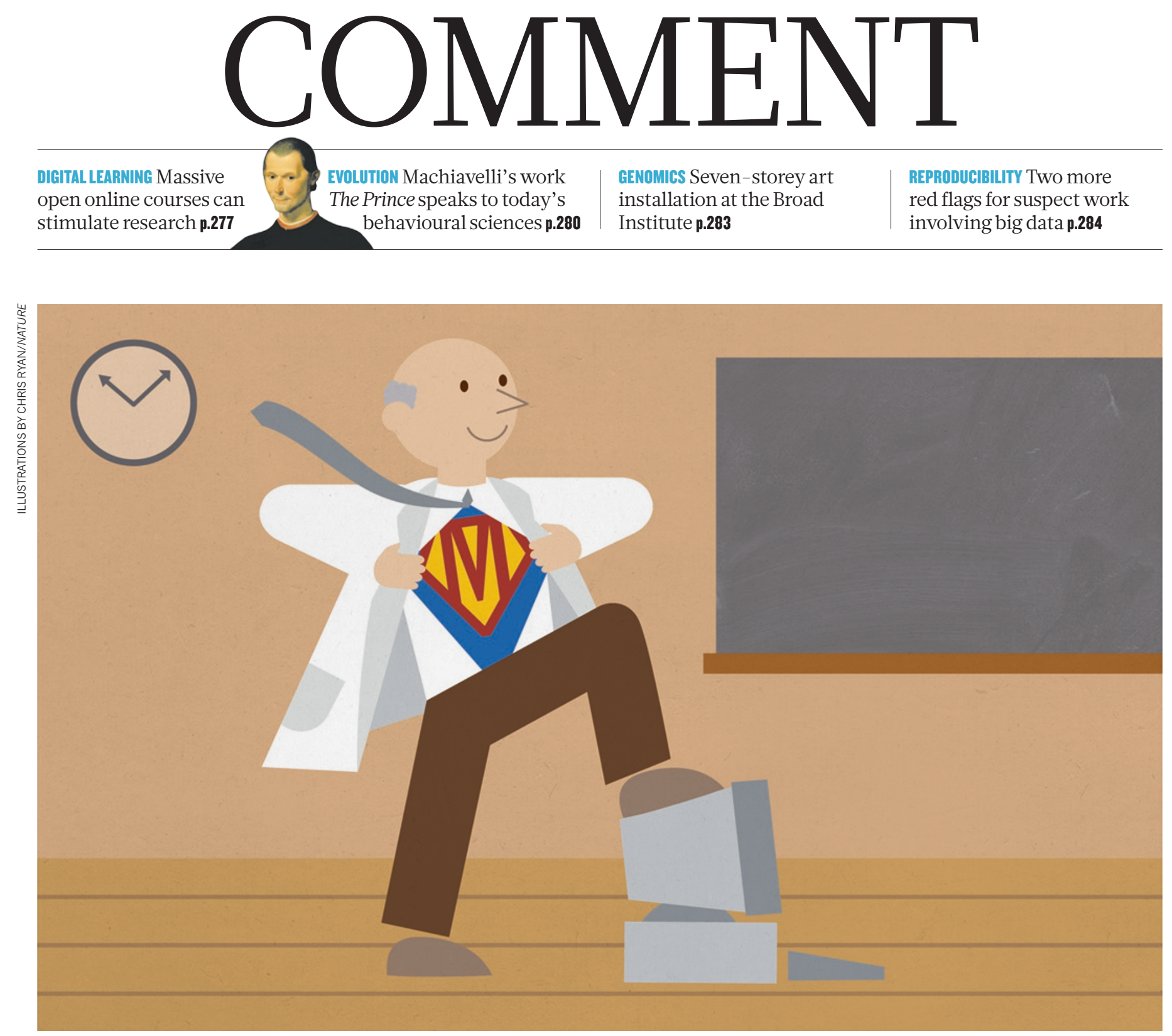

\title{
Look, then leap
}

Massive open online courses can make higher education more accessible, immersive and comprehensive - if they are deployed with due caution, says Michael M. Crow.

$\mathrm{T}$

The response by many in academia and the media to massive open online courses (MOOCs) that make content freely accessible to millions of learners is reminiscent of the hysteria with which alien invaders were met in the pulp science-fiction films of the 1950s. Folks fear the unknown.

More reasoned reception was expressed in a highly publicized open letter sent in April from members of the philosophy department at San José State University in California to Michael Sandel, a professor of government at Harvard University in Cambridge, Massachusetts (see go.nature. com/7n9tgi). The letter explained a refusal to pilot Sandel's interactive online course on social justice. The 'blended' course, which combined online learning with classroom teaching, was proffered by edX, the non-profit

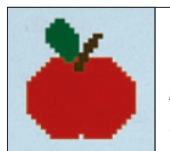

provider of MOOCs established by Harvard and the Massachusetts Institute of Technology in Cambridge. The San José philosophers stated that the "move to MOOCs comes at great peril to our university" and threatens "serious compromise of quality of education". In May, 58 members of the Harvard University Faculty of Arts and Sciences expressed similar scepticism over MOOCs in a letter to their dean, saying that they were "deeply concerned" about the "impact online courses will have on the higher education system as a 


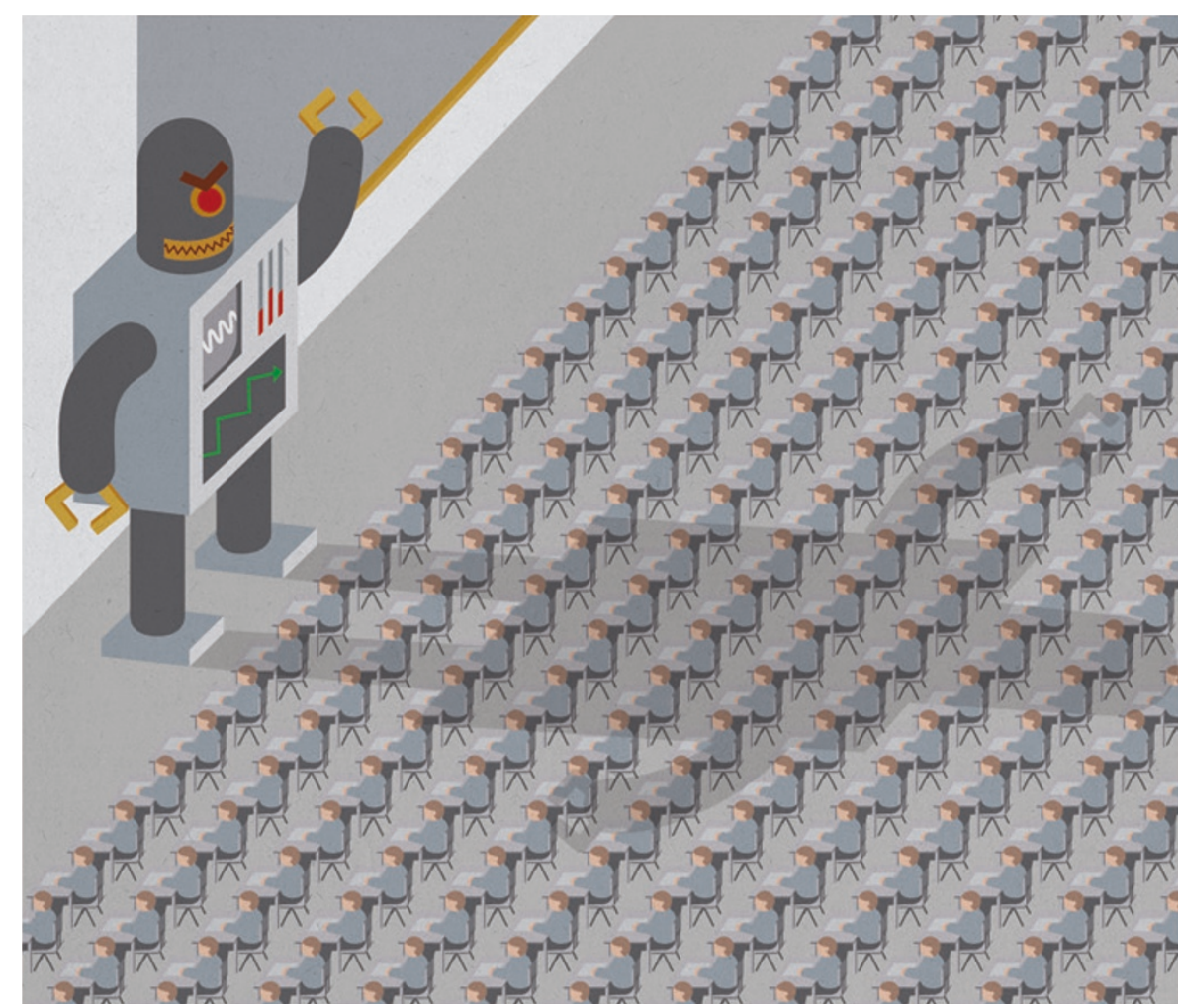

whole" (see go.nature.com/tiru87).

What these letters identify is the lingering ambivalence in academia towards the transition from teaching and learning, based on the fifteenth-century technology of the printed word, to twenty-first-century interactive technologies that offer the potential for adaptive, personalized learning on an infinite scale. Society has embraced the Internet as axiomatic to individualized learning, personal empowerment, cultural evolution and, more recently, revolution. People born after 1990 have never known life without the Internet; for them, it is as integral to learning as it is to their social lives. All aspects of education - from information acquisition to adaptive learning - have been forever altered by a technology that only 20 years ago was considered by nationally prominent academics to be a passing craze.

The pace of technological change has generated much soul-searching about how Internet-delivered courses will change the art of learning. Some worry that a single professor on a computer will, for instance, teach all of the microeconomics courses in an entire country. Others imagine that a latter-day Charles Darwin will, by his or her genius, transform the teaching of introductory university-level biology. But the art of learning has already changed completely, because for almost a decade students have had instant access to unlimited information from anywhere or anyone in the world. This has altered all assumptions about academic hierarchy, charismatic authority, pedagogical processes and the structure of the learning community.

Those in academia are still processing the implications of the impact of digital learning on the diverse arena of higher education, in which students from every socioeconomic background attend institutions that range from community colleges to the world's leading research universities. As colleges and universities educate leaders and experts

\section{"We must lead and shape this revolution, not recoil from it, if we are to avoid stifling innovation."} in every sphere of human endeavour and conduct a large share of the world's scientific research, we must lead and shape this revolution, not recoil from it, if we are to avoid stifling innovation.

\section{FROM FAD TO REVOLUTION}

MOOCs have been on the horizon for two decades. In 1993, when I was a faculty member and vice-provost for research at Columbia University in New York, I hosted a meeting for visitors from the University of Illinois who had developed a tool to link computers on different systems and in different places. One of the visitors was Marc Andreessen, then a student and technical assistant. Andreessen went on to co-develop Mosaic, the first widely popular Web browser, which helped to facilitate the Internet boom of the 1990s. Most of the Columbia faculty members attending the presentation dismissed the Internet as a fad. A few of us suspected that this technological innovation might empower and accelerate the creation and dissemination of knowledge.
During the next few years, I oversaw investment by Columbia University in dozens of faculty groups focused on digital learning. In 2000, we launched Fathom.com, an interactive platform offering free academic content and hundreds of courses provided by a consortium of around 15 of the world's leading universities and cultural institutions - arguably an early precursor to MOOCs. By 2002, around 45,000 students had registered for one of Fathom's online courses.

The platform remained online for roughly three years, but it was slow to generate revenue, heavily constrained by the bandwidth limits of the Internet at the time and by cultural confusion as to its purpose, even among members. After all, wasn't learning supposed to be conducted face to face, in environments rich with discourse? Back then, this slow, choppy and often unreliable thing called the Internet lacked sufficient interactive capacity.

Two decades on from that meeting at Columbia, and more than ten years on from Fathom's launch, it baffles me that a similar scepticism of Internet-enhanced learning still thrives among some faculty members, even as some of the world's leading universities embrace online distance learning as a useful platform and millions of learners worldwide enrol in MOOCs. But an online course developed by faculty members and offered through an online degree programme is not the same thing as a MOOC, and we must observe this distinction if we are to gain understanding about their impact.

For students fortunate enough to enrol in research universities and liberal-arts colleges, undergraduate educations are best advanced in a milieu of discovery and learning immersion. If conceived and executed properly, online courses could provide an important complement. For other students - those living in isolated areas, say, or studying later in life - online courses or fully online degree programmes offer an alternative to the traditional undergraduate experience. Notwithstanding that a course is not a curriculum, that a curriculum is not a degree, and that a degree does not automatically produce an enabled and adaptive learner, our evolving capacity to improve on the textbooks of the past is as good for the faculty members and students of a community college as it is for those in leading universities.

The revolutionary aspect of MOOCs is their potential to reach millions of learners who are not enrolled in colleges and universities. It seems ironic that the very institutions that have become the most exclusive in terms of admissions practices and influential in terms of research have taken the lead in offering MOOCs developed by their professors to millions of students. In fact, this transition from limited access to mass-market is consistent with these universities' values: it 
expands their societal impact. I believe that online learning will enable the creation of high-speed and possibly more efficacious multi- and interdisciplinary teaching environments around the world.

\section{CASE BY CASE}

As president of Arizona State University (ASU) - a large public research university that has four campuses in the Phoenix metropolitan area - I am optimistic about the potential for online learning to enable broader access to the highest levels of academic excellence. The ASU has embraced online learning and offers fully online undergraduate and graduate degree programmes. Faculty members are in charge of course content and complement online instruction with personal interaction. Interactive, adaptive learning platforms break down content and present it to students at an optimal pace and in a tailored sequence, while giving detailed feedback on performance to both students and professors. Our objective is to create an environment in which a person can learn anything, and thus we must avail ourselves of every tool, mechanism and means. The challenge remains for the ASU, like all universities, to evaluate the relevance of MOOCs to curricula and degree programmes and to determine whether and how to proceed with their implementation. This will inevitably advance on a case-by-case basis.
Regardless of any apprehension about online learning, there is now an opportunity to transcend the limits of the 'sage on the stage'. Online learning - including MOOCs - can enable and accelerate deeper or broader learning in certain subjects and thus

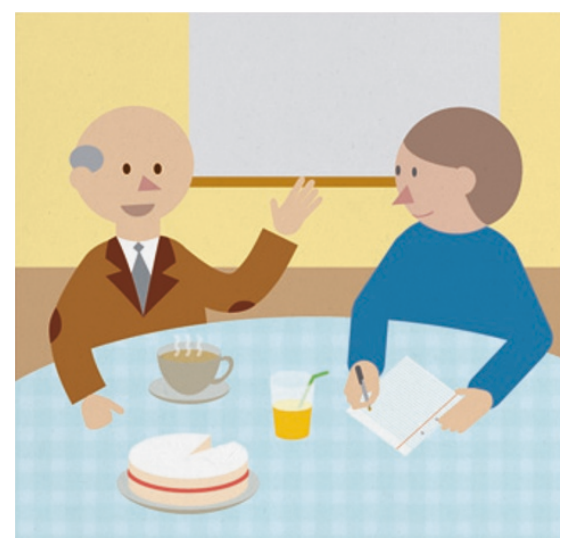

complement face-to-face or team-learning modalities. Where appropriate, MOOCs represent a simple, straightforward way to put quality content in front of lots of students at the same time, enabling scarce resources to be deployed where they are most needed: in the creation of teaching, learning and discovery environments that can educate millions to the levels necessary to ensure successful and continued adaptation in the future.
One cautionary note should be sounded: to the naive, MOOCs seem like the quick fix for cost containment - itself a crucial objective if there is to be any social equity in the higher-education system. A professor from an exclusive university, such as Columbia, could have his or her course put onto the Web as a MOOC, then students at larger public universities could take that course instead of its equivalent on their home campuses, thereby shrinking faculties and costs. But producing a master adaptive learner will always require interaction with the creative and caring teacher-scholars known as the faculty. The integration of interactive online learning tools such as MOOCs can be useful, but these disembodied teaching vehicles are no substitute for teacher interaction.

I can only hope that in our enthusiasm for MOOCs, we look before we leap into a dystopia in which the masses are taught by robots, and the gifted and wealthy by living, breathing professors. We need to end up in a world in which technology once and for all makes higher education more accessible by lowering its cost and enhancing its impact across our great and highly diverse society.

Michael M. Crow is president of Arizona State University, Tempe, Arizona 85287, USA. He previously served as executive vice-provost and professor of science policy at Columbia University in New York. e-mail:michael.crow@asu.edu

\section{Online on-ramps}

\section{Giving scientists greater access to conceptual and technical tuition through massive open online courses will aid interdisciplinary research, say Hazel Sive and Sanjay Sarma.}

$\mathrm{T}$ here has been a lot of discussion about the way in which massive open online courses (MOOCs) might change the landscape of education. A transformation is likely, but in a form more nuanced than current conversations imply (see go.nature. com/cynqvp).

Another question has had less attention: how will MOOCs affect research and scholarly enterprise at universities? Will the courses lead to a decline in the amount or quality of study? Or will a huge increase in online learning improve research?

We are both deeply involved in the MOOC culture at the Massachusetts Institute of Technology (MIT) in Cambridge. We are members of the university's Task Force on the Future of MIT Education, which focuses on the use and effect of MOOCs, and we both run active research groups, so we appreciate the dual tugs of teaching and scholarship. Our opinion is that, at least for residential, research-focused universities, the main impact of MOOCs on research culture will be to aid interdisciplinary work by enabling greater access to conceptual or technical tuition.

Many factors influence scholarship and research in a university. These include funding, space, equipment, research collaborations, the number of faculty members, the

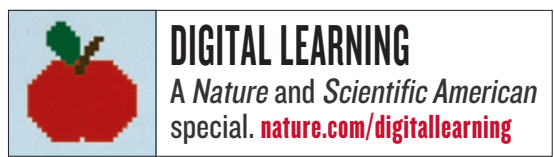

presence of faculty members on campus and the time available for research. Research is often deeply affected by the insight and questioning of smart undergraduate students or postdocs. Fields move forwards through the ability to think in novel, cross-disciplinary ways, and through familiarity with state-ofthe-art techniques.

Which parts of that equation will MOOCs affect? A common view is that they will replace professors. Clearly, this would diminish the volume of research and stifle the collegial discourse that fosters collaboration and original thinking. We do not consider this to be a likely outcome - except perhaps in parts of the world where students have limited access to quality education and where a MOOC might stand in for 